\title{
Elasticity of magnesite and dolomite from a genetic algorithm for inverting Brillouin spectroscopy measurements
}

\author{
Po-Fei Chen ${ }^{\mathrm{a}, *}$, Ling-Yun Chiao ${ }^{\mathrm{b}}$, Pao-hsien Huang ${ }^{\mathrm{a}, \mathrm{b}}$, \\ Yi-jong Yang ${ }^{\text {a }}$, Lin-gun Liu ${ }^{\text {a }}$ \\ a Institute of Earth Sciences, Academia Sinica, Taipei, Taiwan \\ ${ }^{\mathrm{b}}$ Institute of Oceanography, National Taiwan University, Taipei, Taiwan
}

Received 17 January 2005; received in revised form 5 September 2005; accepted 7 October 2005

\begin{abstract}
A hybrid numerical scheme that simultaneously retrieves single-crystal elastic constants, $C_{i j}$, and wave-normal directions is applied to determine the elasticity of natural samples of both magnesite and dolomite, as measured by Brillouin spectroscopy at ambient conditions. The scheme incorporates the genetic algorithm (GA) as a global searching tool and a final local linearization to overcome the intrinsic difficulty of fitting non-linear Christoffel's equation. To compensate for the stochastic nature of GA, especially when the misfit function of the problem manifests highly rugged topography in the model space, the procedure was repeated for multi-runs. This is to assure that the best solution is captured by examining the statistics among the runs that yield the fittest solutions. The resultant elastic constants $C_{11}, C_{12}, C_{13}, C_{14}, C_{33}$ and $C_{44}$ are, respectively, 260.3(2.6), 82.9(4.5), 59.6(3.1), (-)20.1(1.3), 153.7(4.1) and 59.7(1.4) $\mathrm{GPa}$ for magnesite. For dolomite, $C_{11}, C_{12}, C_{13}, C_{14}, C_{15}, C_{33}$ and $C_{44}$ are, respectively, 204.1(2.2), 68.5(3.4), 45.8(4.4), 20.6(1.3), 6.7(1.5), 97.4(5.3) and 39.1(1.5) GPa. Unfortunately, the results for dolomite are statistically non-unique in the numerical calculation. The above data were adopted because the cleavage plane of dolomite was used in the experiment. The results for both magnesite and dolomite are compatible with those determined earlier by ultrasonic methods. It is anticipated that the new method developed in the present study should be applicable to less symmetric crystals, since there are no particular assumptions on the crystal symmetry embedded within the scheme.
\end{abstract}

(c) 2005 Elsevier B.V. All rights reserved.

Keywords: Elasticity; Magnesite; Dolomite; Genetic algorithm

\section{Introduction}

Brillouin spectroscopy determines velocities of acoustic waves traveling along certain directions in a single crystal through measurements of the frequency

\footnotetext{
* Corresponding author. Present address: Institute of Geophysics, National Central University, Chung-Li, Taiwan 320, Taiwan, ROC. Tel.: +886 3 4227837; fax: +886 34222044 .

E-mail address: bob@geps.gep.ncu.edu.tw (P.-F. Chen).
}

shifts of light scattered from the wave phonons (e.g., Sinogeikin and Bass, 2000). Because it requires only small samples and employs visible light, the technique is easily implemented with a diamond-anvil cell to determine the elasticity of minerals at high pressures and/or high temperatures (Duffy et al., 1995; Zha et al., 1998).

In order to compute elastic constants of a crystal from measured acoustic velocities, one has to employ Christoffel's equation (see below) that was derived from a plane-wave solution of the wave equation for 
anisotropic media and that relates the elastic constants and wave vector to the acoustic velocities and density (Auld, 1973; Anderson, 1989). However, the coupling of elastic constants and direction cosines of wave vectors in Christoffel's equation makes inverting elastic constants from acoustic velocities essentially a complex non-linear problem, which has, to some extent, limited the application of the technique to the study of elasticity of crystals. If direction cosines are not previously determined from X-ray analysis, the non-linear inversion is conventionally solved by a two-stage optimization procedure, which iteratively inverts direction cosines and elastic constants back and forth (Every, 1980; Every and Sachse, 1990). The approach is in essence a local linearization scheme that might suffer from entrapment within a local minimum and/or slow convergence. We, therefore, explore the established efficient global searching tool, the genetic algorithm (GA) (Holland, 1975), for inverting Brillouin spectroscopy measurements in this study.

Carbonates possessing the calcite-type structure (space group $R 3 c$ ) are an important group of minerals that have attracted many experimental and theoretical studies (e.g., Reeder, 1983). Among them, calcite $\left(\mathrm{CaCO}_{3}\right)$ and magnesite $\left(\mathrm{MgCO}_{3}\right)$ are the type of alkaline-earth carbonates that have been widely studied. Dolomite $\left(\mathrm{CaMgC}_{2} \mathrm{O}_{6}\right)$ is a half calcium and half magnesium variant, which results in a slightly lower symmetry (space group $R 3$ ). All three carbonates belong to the trigonal system. While calcite and magnesite require six elastic constants $\left(C_{11}, C_{12}, C_{13}, C_{14}, C_{33}\right.$ and $\left.C_{44}\right)$ to describe completely their elastic properties, the lower symmetry of dolomite takes an additional $C_{15}$ to completely describe its elasticity. Chen et al. (2001) have demonstrated that the elastic constants of these three carbonates are inversely proportional to the unit cell volume. However, while the singlecrystal elastic properties of calcite have been extensively studied both by ultrasonic techniques (Dandekar, 1968a,b; Hearmon, 1979; Vo Thanh and Lacam, 1984) and by Brillouin spectroscopy (Chen et al., 2001), only one set of ultrasonic data each for dolomite (Humbert and Plique, 1972) and magnesite (Hearmon, 1979) is available in literature. In this study, Brillouin spectroscopy of magnesite and dolomite at ambient conditions is performed and GA is applied to globally search the potential model space of both elastic constants and direction cosines of wave vectors simultaneously. The neighborhood of the global minimum rapidly located by GA is then swept with a local linearization scheme as an efficient targeting procedure. The hybrid algorithm proved to be an effective approach for retrieving elastic constants of magnesite and dolomite.

\section{Experimental procedures}

\subsection{Samples}

The single crystals of dolomite were provided by T.P. Mernagh from Geoscience Australia. The magnesite crystals were cut from a sample excavated from Liao-ning province, China. Both samples were identified by Raman spectra and the latter was also confirmed by an X-ray diffraction study and its chemical composition was determined by an electron microprobe. The dolomite sample is already a thin platelet $(\sim 200 \mu \mathrm{m}$ in thickness) with roughly parallel cleavage planes. The cutlet planes of magnesite were abraded and polished to parallel each other within $10^{\prime}$ and to a thickness of $\sim 200 \mu \mathrm{m}$.

\subsection{Brillouin scattering experiment}

An argon ion laser $(\lambda=514.5 \mathrm{~nm})$ and a six-pass tandem Fabry-Pérot interferometer were used for the Brillouin experiments. A platelet of each sample was mounted on a three-circle Eulerian cradle, which was used to control the sample with different co-planar orientations. A symmetric scattering geometry was employed with an external angle of $90^{\circ}$ between the incident and scattered beams. With this geometry, the refractive index can be cancelled out in the calculation of acoustic velocities and the Brillouin frequency shift $\Delta \omega$ is directly related to the acoustic velocity $V$ and incident laser wavelength $\lambda$ by:

$V=\frac{\Delta \omega \lambda}{\sqrt{2}}$

A Brillouin spectrum consists of an elastically scattered component with the same frequency as the excitation source and a set of inelastically scattered components. The latter displays a frequency shift caused by the interaction between photons and phonons in the sample. Our Brillouin spectra were collected from the platelet on an arbitrary position rotating from $0^{\circ}$ to $180^{\circ}$ with a $10^{\circ}$ interval. At each angle, the spectra were recorded from at least three different positions on the same plane and then the average was taken to determine the acoustic velocities. Details of the Brillouin scattering technique have been elucidated earlier (e.g., Sinogeikin and Bass, 2000; Chen et al., 2001). 


\section{Inverting for the elastic constants}

\subsection{Forward formulations}

Christoffel's equation is as follows (Anderson, 1989):

$\Lambda_{i j}-\delta_{i j} \rho V^{2}=0$

where $\Lambda_{i j}$ are functions of the elastic constants and direction cosines of wave vector, $\delta_{i j}$ the Kronecker delta, $\rho$ the density and $V$ is the acoustic velocity. When the cradle is rotated at an arbitrary angle $\theta$ (i.e., $0^{\circ}, 10^{\circ}, 20^{\circ}$, $\ldots$..), we denote the direction cosines of the wave vector described by crystallographic coordinates as $l_{\theta}, m_{\theta}$ and $n_{\theta}$. For dolomite, one has:

$$
\begin{aligned}
\Lambda_{11}= & l_{\theta}^{2} C_{11}+m_{\theta}^{2}\left(\frac{C_{11}-C_{12}}{2}\right) \\
& +n_{\theta}^{2} C_{44}+2 m_{\theta} n_{\theta} C_{14}+2 n_{\theta} l_{\theta} C_{15} \\
\Lambda_{22}= & l_{\theta}^{2}\left(\frac{C_{11}-C_{12}}{2}\right)+m_{\theta}^{2} C_{11} \\
& +n_{\theta}^{2} C_{44}-2 m_{\theta} n_{\theta} C_{14}-2 n_{\theta} l_{\theta} C_{15} \\
\Lambda_{33}= & \left(l_{\theta}^{2}+m_{\theta}^{2}\right) C_{44}+n_{\theta}^{2} C_{33} \\
\Lambda_{21}= & -2 m_{\theta} n_{\theta} C_{15}+2 n_{\theta} l_{\theta} C_{14} \\
& +l_{\theta} m_{\theta}\left(\frac{C_{11}+C_{12}}{2}\right) \\
\Lambda_{31}= & \left(l_{\theta}^{2}-m_{\theta}^{2}\right) C_{15}+n_{\theta} l_{\theta}\left(C_{13}+C_{44}\right)+2 l_{\theta} m_{\theta} C_{14} \\
\Lambda_{32}= & \left(l_{\theta}^{2}-m_{\theta}^{2}\right) C_{14}+m_{\theta} n_{\theta}\left(C_{13}+C_{44}\right)-2 l_{\theta} m_{\theta} C_{15} \\
\Lambda_{12}= & \Lambda_{21} \\
\Lambda_{13}= & \Lambda_{31} \\
\Lambda_{23}= & \Lambda_{32}
\end{aligned}
$$

For magnesite, one simply assumes $C_{15}=0$ in Eq. $\left(2^{\prime}\right)$. According to Eq. (2), the three eigenvalues of $\Lambda_{i j}$ correspond to $\rho V^{2}$, where $V$ corresponds to the compressional wave velocity $\left(V_{p}\right)$ or one of the two transverse wave velocities $\left(V_{s 1}\right.$ and $\left.V_{s 2}\right)$ as measured and derived from Brillouin frequency shifts. Because the cradle was rotated from $0^{\circ}$ to $180^{\circ}$ with a $10^{\circ}$ interval, there are 19 sets of $\left(l_{\theta}, m_{\theta}, n_{\theta}\right)$ corresponding to 19 sets of determined $\left(V_{p}, V_{s 1}, V_{s 2}\right)$, while $C_{i j}$ 's remain unchanged. Nevertheless, the 19 sets of $\left(l_{\theta}, m_{\theta}, n_{\theta}\right)$ are not completely independent because they are rotated about the same axis (normal to the platelet). The general Cartesian rotation matrix is (Boisen and Gibbs, 1985):

$$
M(\phi)=\left[\begin{array}{lll}
n_{1}^{2}(1-c \phi)+c \phi & n_{1} n_{2}(1-c \phi)-n_{3} s \phi & n_{1} n_{3}(1-c \phi)+n_{2} s \phi \\
n_{2} n_{1}(1-c \phi)+n_{3} s \phi & n_{2}^{2}(1-c \phi)+c \phi & n_{2} n_{3}(1-c \phi)-n_{1} s \phi \\
n_{3} n_{1}(1-c \phi)-n_{2} s \phi & n_{3} n_{2}(1-c \phi)+n_{1} s \phi & n_{3}^{2}(1-c \phi)+c \phi
\end{array}\right]
$$

where $c \phi=\cos \phi$ and $s \phi=\sin \phi$. Eq. (3) relates the new wave vector to the old one upon rotation of $\phi$ degree about the unit vector $\left(n_{1}, n_{2}, n_{3}\right)$ described by Cartesian coordinates. To simulate the experiments, the Cartesian coordinates are taken as the crystallographic coordinates. Therefore, the wave vector of cradle at angle $\theta$ can be derived from the $0^{\circ}$ wave vector through (taking $\theta=80^{\circ}$ as an example):

$$
\left[\begin{array}{c}
l_{80} \\
m_{80} \\
n_{80}
\end{array}\right]=M(-80)\left[\begin{array}{c}
l_{0} \\
m_{0} \\
n_{0}
\end{array}\right] \text {. }
$$

It is the crystallographic coordinates that rotate when we rotate the cradle, so a negative sign arises in the rotation matrix. If $n_{1}$ and $n_{2}$ are known, $n_{3}$ can be derived from $n_{1}^{2}+n_{2}^{2}+n_{3}^{2}=1$ and a vector on the plane of platelet can be created as $\left(n_{2},-n_{1}, 0\right)$. If the angle between $\left(l_{0}\right.$, $\left.m_{0}, n_{0}\right)$ and $\left(n_{2},-n_{1}, 0\right)$ is $\eta$, we have:

$\left[\begin{array}{c}l_{0} \\ m_{0} \\ n_{0}\end{array}\right]=M(\eta)\left[\begin{array}{c}n_{2} \\ -n_{1} \\ 0\end{array}\right]$.

In other words, all the 19 sets of $\left(l_{\theta}, m_{\theta}, n_{\theta}\right)$ are functions of $n_{1}, n_{2}$ and $\eta$, exclusively. We end up inverting for the six $C_{i j}$ 's and the three variables $\left(n_{1}, n_{2}, \eta\right)$ representing crystal orientation.

\subsection{Brief introduction to the genetic algorithm (GA)}

The genetic algorithm is a numerical optimization scheme that mimics the biological evolution process. It proceeds with a chromosomal representation of model parameters. An initial population of probable solutions is randomly generated within the model space, which is then followed by crossover to produce the next generation solutions with higher probability assigned to those with lower misfits of the cost function. Since better solutions have a stronger resemblance to the best solution in a chromosomal representation, the segment pattern of the best solution tends to be passed to next generation, as attested by reduction of overall misfit. To avoid being trapped within a local minimum for highly rugged model space topography, a probability of mutation is assigned to expand sampling of the model space. After generations of repeating the crossover and mutation, the solution population eventually converges to the best solution, a 

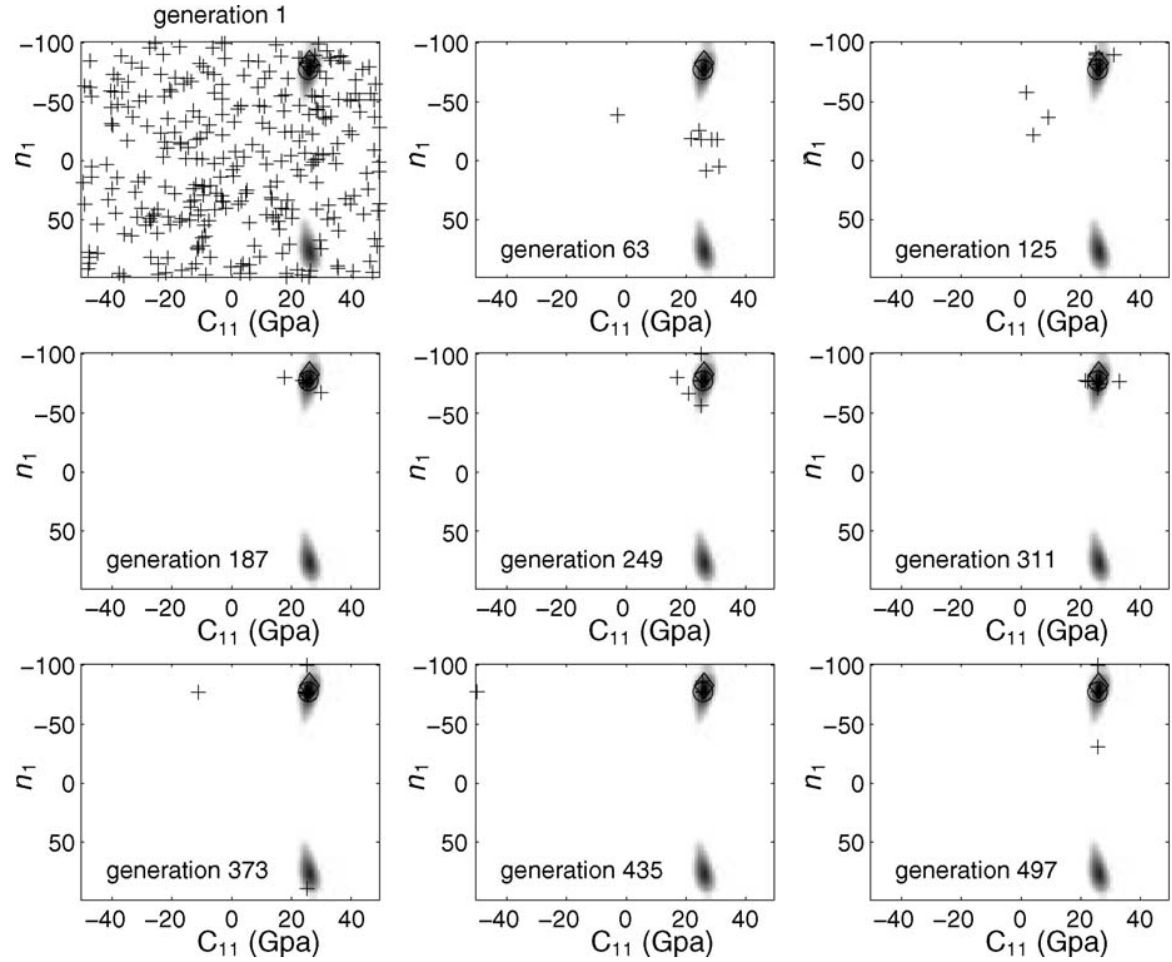

Fig. 1. Distribution of population (cross sign) in different generations as projected on $C_{11}$ (horizontal axis, divided by 10) and $n_{1}$ (vertical axis, multiplied by 100) subspace. The gray area is the regional topography low (least misfit) as calculated from forward formulation, using the best solution found by GA (circle). The final solution is further fine-tuned by local linearization (diamond). Note the local minimum in the lower part of all figures.

mathematical equivalent of "the survival of the fittest" (Michalewicz, 1996). Notably, GA is a global minimum searcher that is much more efficient than a simple brute force grid search which is usually impractical for the model space with high dimensions. However, due to the stochastical nature of the setup of the initial solutions population, the solution from GA is not necessarily the best solution achievable. In this study, we overcome this problem by multi-runs (discussed later).

\subsection{Implementations}

Christoffel's equation is able to predict the measured velocity if we have the correct elastic tensor and the crystal orientation. We adopt a Matlab implementation of GA (Houck et al., 1995) for inversion of the observed acoustic velocities. The model parameters to be inverted are elastic parameters $\left(C_{11}, C_{12}, C_{13}, C_{14}, C_{33}\right.$ and $\left.C_{44}\right)$ for magnesite or $\left(C_{11}, C_{12}, C_{13}, C_{14}, C_{15}, C_{33}\right.$ and $\left.C_{44}\right)$ for dolomite and the parameters $\left(n_{1}, n_{2}\right.$ and $\left.\eta\right)$ specifying the crystal orientation. The unit system was empirically chosen to be $10 \mathrm{GPa}$ for $C_{i j}, 10 \mathrm{~g} / \mathrm{cm}^{3}$ for $\rho$ and $\mathrm{km} / \mathrm{s}$ for wave velocity. Consequently, the bounds of the potential solution space are $(-50,50)$ for $C_{i j},(-1,1)$ for $n_{1}$ and $n_{2}$, and $\left(0^{\circ}, 180^{\circ}\right)$ for $\eta$. We set the initial population size of GA to be 300 . The density $\rho$ is $3.00 \mathrm{~g} / \mathrm{cm}^{3}$ for magnesite and $2.85 \mathrm{~g} / \mathrm{cm}^{3}$ for dolomite. For each member in the population, the 19 sets of $\left(l_{\theta}, m_{\theta}, n_{\theta}\right)$ were calculated from $n_{1}, n_{2}$ and $(\eta-\theta)$ according to Eqs. (4) and (5) and

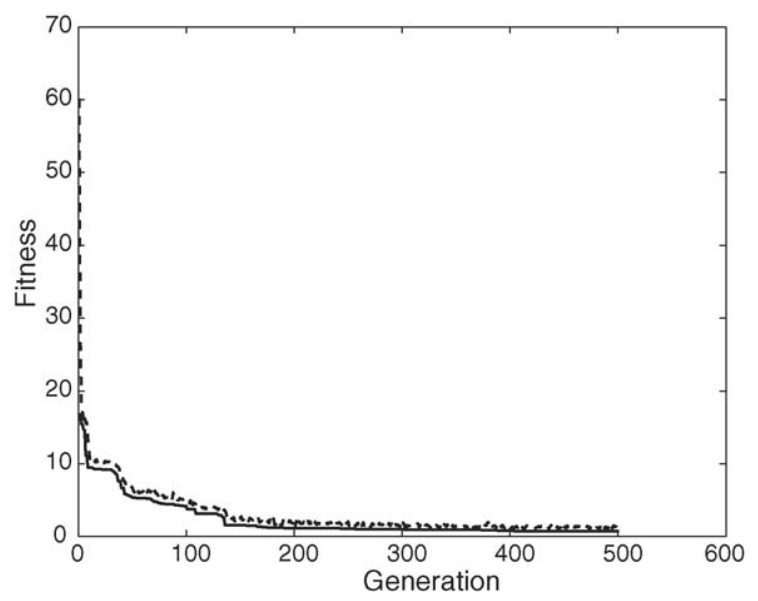

Fig. 2. Fitness vs. generation plot. The dashed line is the average of all individuals in the population at a specific generation, whereas the solid line is the one with least misfit. Both lines demonstrate that the improvement of fitness is limited after 200 generations. 
then inserted into $\Lambda_{i j}$ to derive the 19 sets of eigenvalues. The composite misfit of the latter together with the 19 sets of observed $\rho V_{p}, \rho V_{s 1}, \rho V_{s 2}$ is evaluated. A new generation is then produced according to the crossover and mutation described above. A total of 500 generations are carried out to locate the global minimum. It is noted that although GA is very effective in locating the neighborhood of the global minimum at an initial stage, the rate of convergence to the global minimum is significantly retarded after a few hundred generations as the population is closely clustered. Therefore, instead of evolving through large number of generations, we incorporate a local search mechanism, e.g., Levenberg-Marquardt scheme (Press et al., 1992), upon the GA solution to pinpoint the global minimum, i.e., an implementation of a hybrid numerical scheme.

The application of GA on magnesite data is elucidated in Figs. 1-3. The effectiveness of the evolution to converge to better solutions in the first few tens of generations is clearly depicted by the distribution and migration of the solutions population from different generations on the projected $n_{1}$ (vertical) versus $C_{11}$ (horizontal)

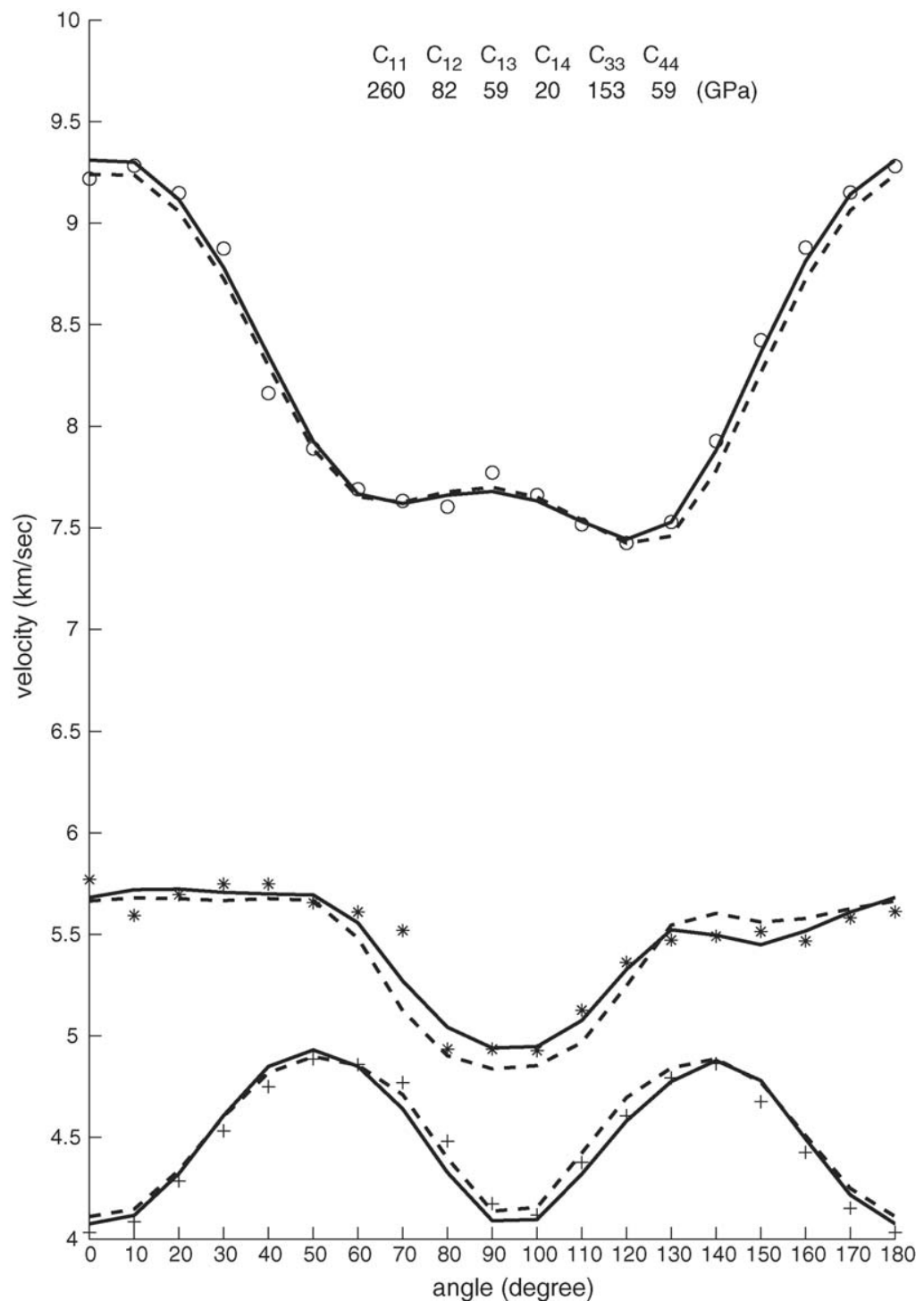

Fig. 3. The experimental data (open circles for longitudinal velocity and stars and crosses for two transverse velocities) are compared with those (solid and dashed lines) calculated from the elastic constants using the $C_{i j}$ 's shown on top, as a function of the rotating angle. The dashed lines are the results of GA application only and the solid lines are the final results after conducting a local linearization at the neighborhood of the solution found by GA. 
Table 1

The best 50 inverted results for magnesite in 200 runs

\begin{tabular}{|c|c|c|c|c|c|c|c|c|c|c|c|c|c|}
\hline Run no. & $C_{11}$ & $C_{12}$ & $C_{13}$ & $C_{14}$ & $C_{33}$ & $C_{44}$ & $n_{1}$ & $n_{2}$ & $\eta$ & Misfit & $\alpha$ & $\beta$ & $\gamma$ \\
\hline 31 & 260 & 83 & 60 & 20 & 153 & 60 & -0.83 & -0.36 & 1.1 & 0.55 & 146.0 & 111.0 & 64.6 \\
\hline 46 & 260 & 83 & 60 & -20 & 153 & 60 & -0.10 & -0.90 & 7.7 & 0.55 & 96.0 & 153.6 & 64.4 \\
\hline 59 & 260 & 83 & 60 & -20 & 153 & 60 & -0.10 & -0.90 & 7.7 & 0.55 & 96.0 & 153.6 & 64.4 \\
\hline 107 & 260 & 83 & 60 & -20 & 153 & 60 & -0.72 & 0.54 & 7.7 & 0.55 & 136.4 & 57.4 & 64.5 \\
\hline 128 & 260 & 83 & 60 & -20 & 153 & 60 & -0.72 & 0.54 & 7.7 & 0.55 & 136.4 & 57.4 & 64.5 \\
\hline 163 & 260 & 83 & 60 & 20 & 153 & 60 & 0.10 & 0.90 & in & 0.55 & 84.0 & 26.4 & 64.4 \\
\hline 173 & 260 & 83 & 60 & 20 & 153 & 60 & 0.72 & -0.54 & 1.1 & 0.55 & 43.6 & 122.6 & 64.5 \\
\hline 21 & 50 & -158 & -202 & -40 & -500 & 250 & -0.39 & -0.32 & 4.5 & 0.60 & 113.1 & 108.5 & 30.4 \\
\hline 154 & 50 & -158 & -202 & 40 & -500 & 250 & 0.08 & -0.50 & 4.5 & 0.60 & 85.5 & 120.0 & 30.4 \\
\hline 8 & 50 & -156 & -202 & 40 & -490 & 252 & -0.39 & 0.33 & 4.6 & 0.61 & 113.1 & 70.7 & 30.9 \\
\hline 28 & 50 & -156 & -202 & -40 & -492 & 252 & 0.09 & 0.50 & 4.6 & 0.61 & 84.8 & 59.7 & 30.9 \\
\hline 41 & 50 & -156 & -202 & -40 & -490 & 252 & 0.09 & 0.50 & 4.6 & 0.61 & 84.8 & 59.7 & 30.9 \\
\hline 73 & 50 & -156 & -202 & -40 & -490 & 252 & 0.39 & -0.33 & 4.6 & 0.61 & 66.9 & 109.3 & 30.9 \\
\hline 74 & 50 & -156 & -202 & -40 & -492 & 252 & 0.09 & 0.50 & 4.6 & 0.61 & 84.8 & 59.7 & 30.9 \\
\hline 98 & 50 & -156 & -202 & -40 & -492 & 252 & 0.39 & -0.33 & 4.6 & 0.61 & 66.9 & 109.3 & 30.8 \\
\hline 47 & 176 & 54 & 71 & -12 & 430 & 64 & 0.57 & 0.29 & 95.8 & 0.62 & 55.1 & 73.2 & 39.9 \\
\hline 66 & 176 & 54 & 71 & 12 & 430 & 64 & -0.57 & -0.29 & 95.8 & 0.62 & 124.9 & 106.8 & 39.9 \\
\hline 122 & 176 & 54 & 70 & -12 & 425 & 64 & -0.04 & -0.65 & 95.8 & 0.62 & 92.0 & 130.3 & 40.4 \\
\hline 167 & 176 & 54 & 71 & -12 & 430 & 64 & -0.54 & 0.35 & 95.8 & 0.62 & 122.4 & 69.5 & 39.8 \\
\hline 40 & 261 & 98 & -43 & 22 & 90 & 64 & 0.64 & -0.38 & 4.9 & 0.62 & 49.9 & 112.2 & 48.3 \\
\hline 93 & 261 & 98 & -43 & 22 & 90 & 64 & 0.64 & -0.38 & 4.8 & 0.62 & 49.9 & 112.3 & 48.4 \\
\hline 124 & 261 & 98 & -43 & -22 & 90 & 64 & 0.65 & 0.37 & 4.8 & 0.62 & 49.5 & 68.4 & 48.3 \\
\hline 137 & 261 & 98 & -43 & -22 & 90 & 64 & -0.01 & -0.75 & 4.8 & 0.62 & 90.3 & 138.3 & 48.3 \\
\hline 179 & 261 & 98 & -43 & 22 & 90 & 64 & -0.65 & -0.37 & 4.8 & 0.62 & 130.5 & 111.6 & 48.3 \\
\hline 147 & 49 & -156 & -188 & -35 & -464 & 252 & -0.46 & -0.25 & 4.5 & 0.63 & 117.6 & 104.8 & 32.0 \\
\hline 183 & 49 & -156 & -188 & 35 & -462 & 252 & -0.01 & -0.53 & 4.5 & 0.63 & 90.6 & 122.0 & 32.0 \\
\hline 187 & 49 & -156 & -188 & -35 & -462 & 252 & 0.45 & -0.27 & 4.5 & 0.63 & 63.1 & 105.9 & 32.0 \\
\hline 85 & 60 & -389 & -128 & 70 & 49 & 121 & -0.09 & -0.94 & 10.2 & 0.65 & 95.3 & 160.1 & 70.8 \\
\hline 169 & 60 & -389 & -128 & 70 & 49 & 120 & -0.09 & -0.94 & 10.2 & 0.65 & 95.3 & 160.1 & 70.8 \\
\hline 2 & 49 & -470 & -108 & -20 & 37 & 101 & -0.72 & -0.39 & 5.3 & 0.65 & 135.9 & 112.7 & 54.6 \\
\hline 30 & 49 & -470 & -108 & 20 & 37 & 101 & -0.69 & 0.43 & 5.3 & 0.65 & 133.9 & 64.6 & 54.7 \\
\hline 57 & 49 & -470 & -108 & 20 & 37 & 101 & 0.72 & 0.39 & 5.3 & 0.65 & 44.1 & 67.3 & 54.6 \\
\hline 88 & 49 & -470 & -108 & -20 & 37 & 101 & -0.72 & -0.39 & 5.3 & 0.65 & 135.9 & 112.7 & 54.6 \\
\hline 151 & 49 & -470 & -108 & 20 & 37 & 101 & 0.72 & 0.39 & 5.3 & 0.65 & 44.1 & 67.3 & 54.6 \\
\hline 164 & 49 & -470 & -108 & 20 & 37 & 101 & -0.69 & 0.43 & 5.3 & 0.65 & 133.9 & 64.6 & 54.7 \\
\hline 111 & 49 & -470 & -108 & 20 & 37 & 101 & 0.72 & 0.39 & 5.3 & 0.65 & 44.1 & 67.3 & 54.6 \\
\hline 100 & 48 & -165 & -202 & -40 & -500 & 247 & -0.42 & -0.26 & 4.8 & 0.71 & 114.7 & 105.0 & 29.4 \\
\hline 4 & 261 & 86 & -145 & 17 & 178 & 59 & 0.85 & -0.48 & 4.8 & 0.75 & 32.1 & 118.8 & 77.0 \\
\hline 15 & 261 & 86 & -145 & -17 & 178 & 59 & 0.01 & -0.97 & 4.8 & 0.75 & 89.6 & 166.9 & 76.9 \\
\hline 27 & 261 & 86 & -145 & 17 & 178 & 59 & -0.84 & -0.49 & 4.8 & 0.75 & 147.2 & 119.5 & 77.0 \\
\hline 101 & 261 & 86 & -145 & -17 & 178 & 59 & -0.85 & 0.48 & 4.8 & 0.75 & 147.9 & 61.2 & 77.0 \\
\hline 139 & 261 & 86 & -145 & -17 & 178 & 59 & -0.85 & 0.48 & 4.8 & 0.75 & 147.9 & 61.2 & 77.0 \\
\hline 189 & 261 & 86 & -145 & -17 & 178 & 59 & 0.01 & -0.97 & 4.8 & 0.75 & 89.6 & 166.9 & 76.9 \\
\hline 130 & 49 & -404 & -113 & -66 & 46 & 123 & 0.80 & -0.50 & 5.7 & 0.78 & 37.3 & 120.1 & 70.1 \\
\hline 133 & 74 & -247 & -25 & 37 & 278 & 68 & -0.01 & -0.95 & 94.4 & 0.84 & 90.9 & 162.6 & 72.6 \\
\hline 33 & 260 & 98 & -45 & 22 & 88 & 65 & 0.67 & -0.33 & 180.0 & 0.87 & 48.3 & 109.4 & 48.0 \\
\hline 37 & 260 & 98 & -45 & -22 & 88 & 65 & 0.05 & -0.74 & 180.0 & 0.87 & 87.4 & 137.9 & 48.0 \\
\hline 68 & 260 & 98 & -45 & -22 & 88 & 65 & -0.67 & 0.33 & 180.0 & 0.87 & 131.7 & 70.6 & 48.0 \\
\hline 87 & 260 & 98 & -45 & -22 & 88 & 65 & 0.62 & 0.41 & 180.0 & 0.87 & 51.7 & 65.8 & 48.0 \\
\hline 99 & 260 & 98 & -45 & -22 & 88 & 65 & 0.05 & -0.74 & 180.0 & 0.87 & 87.4 & 137.9 & 48.0 \\
\hline
\end{tabular}


subspace (Fig. 1), as well as the variation of the fitness through generations (Fig. 2). Although the improvement of fitness is limited after a few hundreds of generations (Fig. 2), the proposed hybrid numerical scheme is able to supplement GA to pinpoint the global minimum and to achieve an optimal fitting of measured data as shown in Fig. 3.

Due to the highly rugged fitness terrain arisen from the non-linearity, there are numerous local minima scattered throughout the model space. With a randomly selected initial population, the converged final solution yielded from the improved GA is still not unique. In other words, different solutions might be obtained from different runs based on the same data set. To compensate for the inevitable stochastic characteristics, we decided to repeat the procedure for multi-runs and the best solutions were determined from those with lowest misfits. In short, this exercise seeks to have statistically sufficient coverage of the model space while keeping a manageable size of the initial population. The exact amount of repeating depends on the complexity of the constraining equation or the ruggedness of the fitness terrain. Our experience on the data of magnesite and dolomite measurements suggested that about 200 trials are enough, i.e., the solution is stable in the sense that the best out of every 200 trials remains the same.

\section{Results and discussion}

Each trial represents a new starting model randomly picked by the GA algorithm. Results of 200 trials are sorted in order according to value of misfit. The misfits are evaluated as data-point (57 in the present experiment) times the root mean squares of the data. The best 50 trials (misfit $\leq 0.87$ ) for magnesite are representatively displayed in Table 1, in which the last three columns are the direction cosines $(\alpha, \beta, \gamma)$ of the plane normal. These correspond, respectively, to the $X, Y$ and $Z$ Cartesian coordinates of the crystal shown in Figs. 4 and 5. There are seven solutions among 200 runs that have the same least misfit (0.55) in Table 1 . The seven solutions have almost the same $C_{i j}$ 's, except the negative sign of $C_{14}$. It is also noticed that the seven solutions have the same $\gamma$ and some have different $\alpha$ and $\beta$. The apparent non-uniqueness with the same least misfit is explained as follows. The key issues that need to be clarified include: (1) why does $C_{14}$ flip sign? (2) Are the differences in direction cosines $(\alpha, \beta, \gamma)$ among the seven solutions related? (3) Are the obtained $C_{i j}$ 's consistent with those from ultrasonic measurements?

Crystals having a trigonal system can also be described in terms of a hexagonal frame (Figs. 4 and 5). It

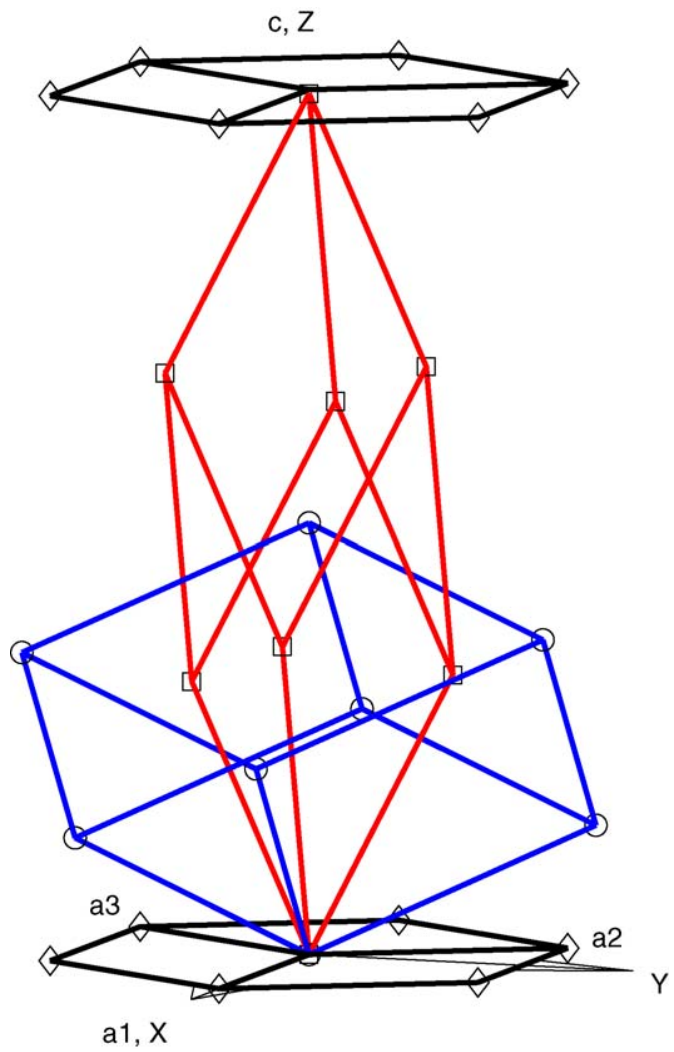

Fig. 4. The calcite-type structure. The red lines and squares depict the unit cell and the blue lines and circles refer to the cleavage rhomb. The black lines and diamonds are the related hexagonal cell with $a$ - and $c$ axes indicated. Also shown are the conventional Cartesian coordinates with respect to the hexagonal system according to Nye (1957).

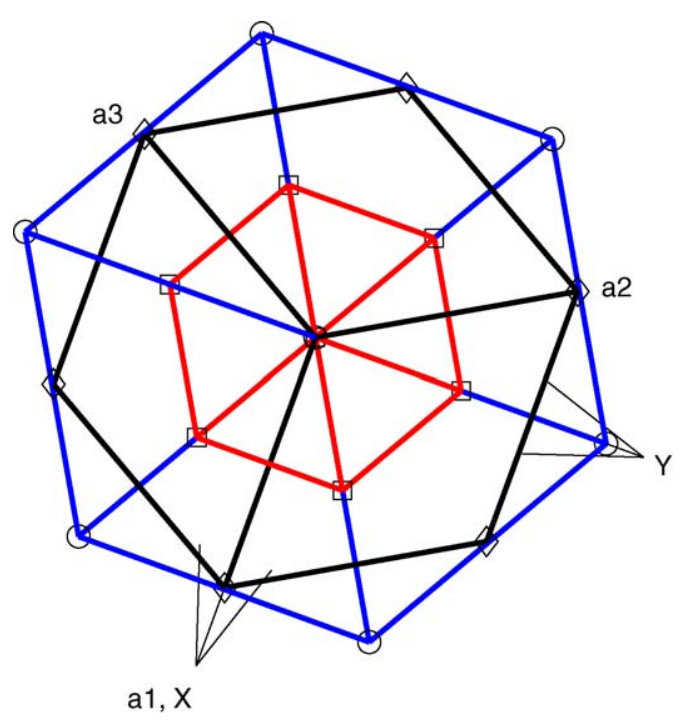

Fig. 5. The top view of Fig. 4. Note that the corners of the unit cell and the cleavage rhomb have the same azimuths and are offset $30^{\circ}$ to the hexagonal axial system. 

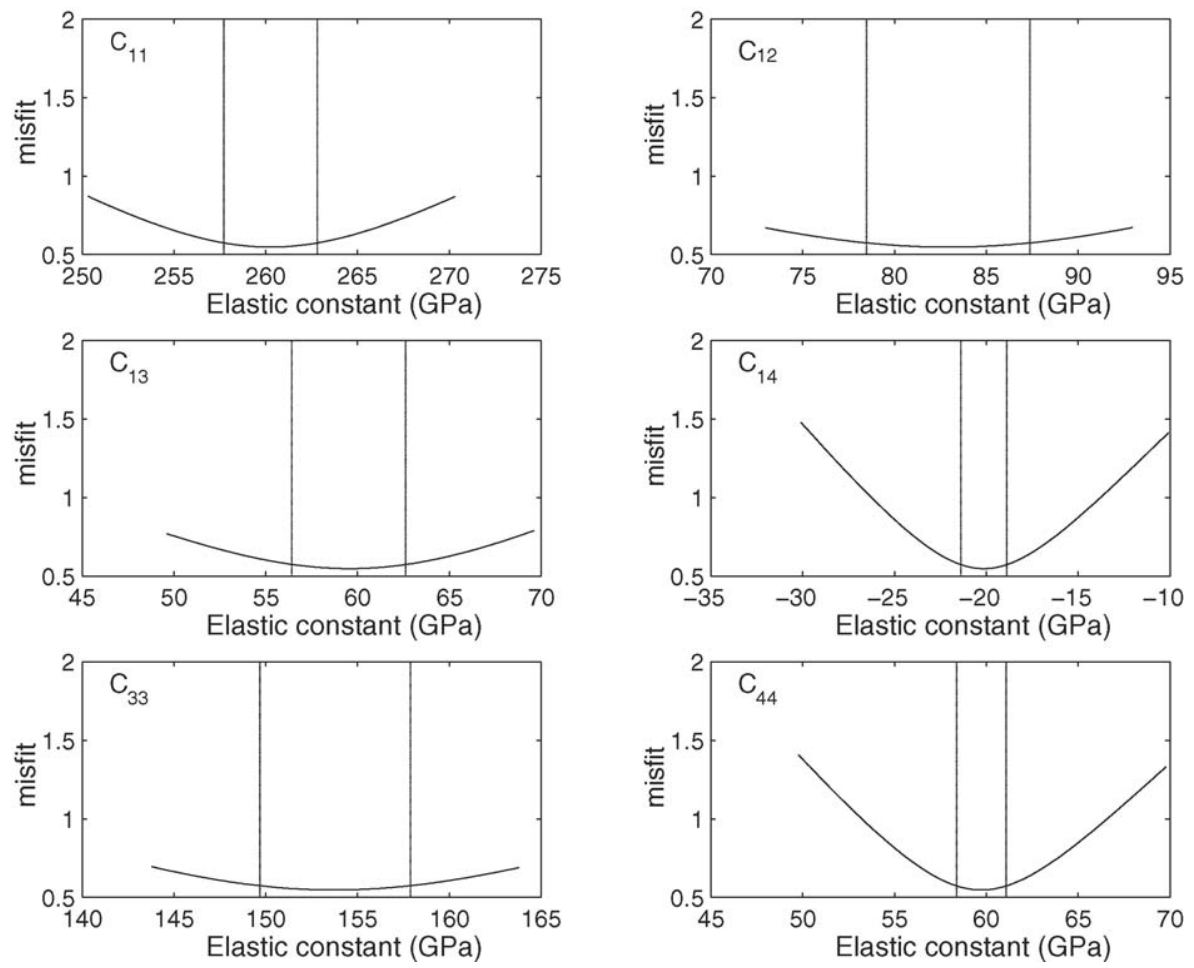

Fig. 6. Variation of misfit as a function of values of elastic constants (in GPa) for magnesite. The two vertical lines in each plot bound the range, where the differential misfit is less than $5 \%$ of the minimum.

is thus a common practice to use the hexagonal description to relate the assigned Cartesian coordinates for $C_{i j}$ 's. According to Nye (1957), the published $C_{i j}$ 's of a hexagonal system refer to the conventional Cartesian coordinates with the $Z$-axis paralleling $c$, the $X$-axis paralleling $a_{1}$, and the $Y$-axis bisecting $a_{2}$ and $-a_{3}$ (Figs. 4 and 5). Bearing this in mind, both questions (1 and 2) can be answered together. The fact that the seven solutions possess nearly the same $\gamma$ angle indicates that the coordinate settings of the solutions may be related by rotating around the $Z$-axis. It is arbitrary to choose the coordinates of Run \#31 as the reference. Then one finds that the coordinates of the other six solutions (Runs \#46, 59, $107,128,163$ and 173) can be, respectively, derived by rotating $60^{\circ}, 60^{\circ}, 300^{\circ}, 300^{\circ}, 240^{\circ}$ and $120^{\circ}$ about the $Z$ axis. Accordingly, a general rule may be established for a trigonal system - the sign of $C_{14}$ changes if the Cartesian reference system is rotated by $(2 n+1) \times 60^{\circ}$ about the $Z$-axis, where $n$ is an integer. Rotations by $2 n \times 60^{\circ}$ leave $C_{14}$ unchanged. Therefore, $C_{14}$ 's of solution Runs \#46, 59, 107 and 128 (rows 2-5, respectively) change sign relative to that of Run \#31, while solution Runs \#163 and 173 (rows 6 and 7, respectively) maintain the same sign. This rule is also observed for solutions with greater misfit in the lower part of Table 1 . The physical explanation is that Christoffel's equations are basically the same for both hexagonal and trigonal systems, except that the latter possesses an extra term of $C_{14}$. This is noticeable that the $Z$-axis symmetry is three-fold for a trigonal system and six-fold for a hexagonal system. Furthermore, it is recalled that $C_{14}$ is either $c_{1123}$ or $c_{1132}$ for a tensor notation. For the purpose of illustration, we visualize $C_{14}$ as the $Z$-direction strain of the surface normal to $Y$ in response to the stresses along $X$-direction. As demonstrated in Figs. 4 and 5, the current dominant plane in the first quadrant of $X-Y$ axes is face down. It is expected that tension along an $X$-direction would induce negative displacements in the $Z$-axis and thus $C_{14}$ is negative. Conversely, the $Z$-direction displacements are positive (positive $C_{14}$ ) in response to the $X$-direction tension for the face-up plane. The latter becomes the dominant plane in the first quadrant of $X-Y$ axes by rotating $(2 n+1) \times 60^{\circ}$ about the $Z$-axis. This explains why rotating $(2 n) \times 60^{\circ}$ does not change the sign of $C_{14}$, but $(2 n+1) \times 60^{\circ}$ does. It is interesting to note that the refinement of elastic constants from measurements highlights the existence of "non-uniqueness" in the choice of a reference system.

The $C_{11}, C_{12}, C_{13}, C_{14}, C_{33}$ and $C_{44}$ for magnesite in this study as determined by the solution of least misfit 
Table 2

The best 50 inverted results for dolomite in 200 runs

\begin{tabular}{|c|c|c|c|c|c|c|c|c|c|c|c|c|c|c|}
\hline Run no. & $C_{11}$ & $C_{12}$ & $C_{13}$ & $C_{14}$ & $C_{15}$ & $C_{33}$ & $C_{44}$ & $n_{1}$ & $n_{2}$ & $\eta$ & Misfit & $\alpha$ & $\beta$ & $\gamma$ \\
\hline 91 & 202 & 118 & 56 & 8 & 22 & 120 & 65 & -0.56 & 0.78 & 36.4 & 0.65 & 124.1 & 38.4 & $\overline{74.6}$ \\
\hline 93 & 202 & 118 & 56 & 22 & 10 & 120 & 65 & -0.75 & 0.61 & 36.4 & 0.65 & 138.4 & 52.6 & 74.6 \\
\hline 104 & 202 & 118 & 56 & -22 & -8 & 120 & 65 & -0.89 & -0.36 & 36.4 & 0.65 & 153.4 & 111.2 & 74.6 \\
\hline 105 & 202 & 118 & 56 & 22 & 10 & 120 & 65 & 0.90 & 0.33 & 36.4 & 0.65 & 25.3 & 70.5 & 74.4 \\
\hline 152 & 202 & 118 & 56 & -12 & -20 & 120 & 65 & -0.95 & -0.14 & 36.4 & 0.65 & 162.6 & 98.0 & 74.6 \\
\hline 199 & 202 & 118 & 56 & 12 & -20 & 120 & 65 & 0.29 & -0.92 & 36.4 & 0.65 & 73.0 & 156.6 & 74.5 \\
\hline 64 & 203 & 68 & 45 & 16 & 15 & 96 & 39 & -0.20 & -0.61 & 38.8 & 0.66 & 101.6 & 127.3 & 39.7 \\
\hline 126 & 203 & 68 & 45 & 6 & -20 & 96 & 39 & -0.63 & 0.11 & 38.8 & 0.66 & 128.9 & 83.5 & 39.6 \\
\hline 156 & 203 & 68 & 45 & 17 & -13 & 96 & 39 & 0.49 & 0.41 & 38.8 & 0.66 & 60.6 & 66.0 & 39.6 \\
\hline 18 & 205 & 150 & -232 & -3 & -8 & 103 & 75 & -0.95 & -0.17 & 36.3 & 0.72 & 161.6 & 99.7 & 74.5 \\
\hline 81 & 205 & 150 & -232 & 8 & -4 & 103 & 75 & -0.91 & 0.32 & 36.3 & 0.72 & 155.4 & 71.3 & 74.5 \\
\hline 31 & 202 & 65 & 55 & 22 & 12 & 141 & 40 & 0.75 & 0.28 & 35.6 & 0.82 & 41.8 & 73.9 & 52.7 \\
\hline 51 & 202 & 65 & 55 & 20 & 13 & 141 & 40 & -0.60 & 0.52 & 35.6 & 0.82 & 126.9 & 58.5 & 52.7 \\
\hline 141 & 202 & 65 & 55 & -20 & 15 & 141 & 40 & -0.17 & 0.78 & 35.6 & 0.82 & 99.9 & 39.0 & 52.7 \\
\hline 6 & 133 & 32 & -257 & 8 & 6 & 182 & 73 & 0.83 & 0.26 & 126.4 & 0.83 & 33.6 & 75.0 & 60.7 \\
\hline 102 & 205 & 148 & 58 & -1 & 11 & 95 & 78 & -0.41 & 0.85 & 36.8 & 0.84 & 114.2 & 32.0 & 70.4 \\
\hline 89 & 202 & 60 & 60 & 22 & -10 & 143 & 37 & 0.66 & 0.42 & 39.5 & 0.85 & 49.0 & 65.2 & 51.1 \\
\hline 21 & 128 & 12 & 105 & 11 & -11 & 231 & 68 & 0.52 & 0.48 & 126.9 & 0.89 & 58.8 & 61.2 & 45.0 \\
\hline 96 & 128 & 12 & 105 & -6 & -13 & 231 & 68 & 0.41 & -0.58 & 126.9 & 0.89 & 66.0 & 125.4 & 45.1 \\
\hline 118 & 128 & 12 & 105 & -6 & -13 & 231 & 68 & 0.42 & -0.57 & 126.9 & 0.89 & 65.2 & 124.7 & 45.0 \\
\hline 119 & 128 & 12 & 105 & 1 & -15 & 231 & 68 & -0.71 & 0.05 & 126.9 & 0.89 & 134.9 & 87.2 & 45.0 \\
\hline 178 & 128 & 12 & 105 & -12 & -10 & 231 & 68 & 0.19 & 0.68 & 126.9 & 0.89 & 78.8 & 47.2 & 45.0 \\
\hline 28 & 70 & -227 & -30 & -11 & -78 & 69 & 91 & -0.96 & -0.15 & 36.4 & 0.89 & 162.9 & 98.8 & 75.5 \\
\hline 174 & 70 & -227 & -30 & 73 & -30 & 69 & 91 & -0.93 & 0.27 & 36.4 & 0.89 & 158.4 & 74.4 & 75.5 \\
\hline 5 & 201 & 108 & 4 & 22 & -6 & 41 & 65 & 0.05 & -0.64 & 37.2 & 0.90 & 87.2 & 130.2 & 40.3 \\
\hline 13 & 201 & 108 & 4 & -12 & -20 & 41 & 65 & -0.64 & -0.10 & 37.2 & 0.90 & 129.7 & 95.8 & 40.3 \\
\hline 19 & 201 & 108 & 4 & -20 & -12 & 41 & 65 & 0.13 & 0.63 & 37.2 & 0.90 & 82.4 & 50.7 & 40.3 \\
\hline 26 & 201 & 108 & 4 & 20 & -10 & 41 & 65 & 0.08 & -0.64 & 37.2 & 0.90 & 85.7 & 130.0 & 40.3 \\
\hline 30 & 201 & 108 & 4 & 20 & 12 & 41 & 65 & -0.13 & -0.63 & 37.2 & 0.90 & 97.7 & 129.3 & 40.3 \\
\hline 37 & 201 & 108 & 4 & 20 & -11 & 41 & 65 & 0.51 & 0.40 & 37.2 & 0.90 & 59.2 & 66.7 & 40.3 \\
\hline 50 & 201 & 108 & 4 & 16 & -17 & 41 & 65 & 0.47 & 0.45 & 37.2 & 0.90 & 62.0 & 63.5 & 40.3 \\
\hline 55 & 201 & 108 & 4 & 4 & -22 & 41 & 65 & -0.64 & 0.06 & 37.2 & 0.90 & 130.2 & 86.7 & 40.4 \\
\hline 62 & 201 & 108 & 4 & -17 & -16 & 41 & 65 & 0.45 & -0.47 & 37.2 & 0.90 & 63.3 & 117.7 & 40.3 \\
\hline 65 & 201 & 108 & 4 & 10 & -20 & 41 & 65 & -0.64 & 0.12 & 37.2 & 0.90 & 129.5 & 83.2 & 40.3 \\
\hline 78 & 201 & 108 & 4 & -22 & -8 & 41 & 65 & 0.09 & 0.64 & 37.2 & 0.90 & 84.8 & 50.1 & 40.3 \\
\hline 92 & 201 & 108 & 4 & -10 & 20 & 41 & 65 & -0.42 & -0.50 & 37.2 & 0.90 & 114.6 & 119.7 & 40.3 \\
\hline 99 & 201 & 108 & 4 & -10 & 20 & 41 & 65 & -0.42 & -0.49 & 37.2 & 0.90 & 114.7 & 119.6 & 40.3 \\
\hline 107 & 201 & 108 & 4 & 22 & -2 & 41 & 65 & 0.00 & -0.65 & 37.2 & 0.90 & 90.2 & 130.3 & 40.3 \\
\hline 113 & 201 & 108 & 4 & 22 & -8 & 41 & 65 & 0.05 & -0.64 & 37.2 & 0.90 & 86.8 & 130.2 & 40.3 \\
\hline 116 & 201 & 108 & 4 & -22 & -2 & 41 & 65 & 0.54 & -0.35 & 37.2 & 0.90 & 57.2 & 110.7 & 40.3 \\
\hline 122 & 201 & 108 & 4 & 2 & -22 & 41 & 65 & -0.65 & 0.04 & 37.2 & 0.90 & 130.2 & 87.8 & 40.3 \\
\hline 132 & 201 & 108 & 4 & -12 & -20 & 41 & 65 & 0.41 & -0.50 & 37.2 & 0.90 & 65.9 & 120.1 & 40.4 \\
\hline 145 & 201 & 108 & 4 & -22 & -6 & 41 & 65 & 0.52 & -0.39 & 37.2 & 0.90 & 58.9 & 112.9 & 40.3 \\
\hline 169 & 201 & 108 & 4 & -5 & -22 & 41 & 65 & -0.65 & -0.03 & 37.2 & 0.90 & 130.3 & 91.5 & 40.4 \\
\hline 173 & 201 & 108 & 4 & 15 & -17 & 41 & 65 & -0.63 & 0.16 & 37.2 & 0.90 & 128.8 & 80.6 & 40.3 \\
\hline 175 & 201 & 108 & 4 & 22 & -6 & 41 & 65 & 0.54 & 0.36 & 37.2 & 0.90 & 57.7 & 68.7 & 40.3 \\
\hline 184 & 201 & 108 & 4 & -3 & -22 & 41 & 65 & 0.33 & -0.56 & 37.2 & 0.90 & 70.5 & 123.7 & 40.3 \\
\hline 190 & 201 & 108 & 4 & -22 & 6 & 41 & 65 & 0.58 & -0.28 & 37.2 & 0.90 & 54.4 & 106.5 & 40.4 \\
\hline 193 & 201 & 108 & 4 & -12 & 20 & 41 & 65 & -0.20 & 0.62 & 37.2 & 0.90 & 101.7 & 52.0 & 40.4 \\
\hline 25 & 79 & -12 & -70 & -11 & -46 & -283 & 187 & 0.24 & 0.46 & 38.2 & 0.99 & 76.1 & 62.5 & 31.4 \\
\hline
\end{tabular}




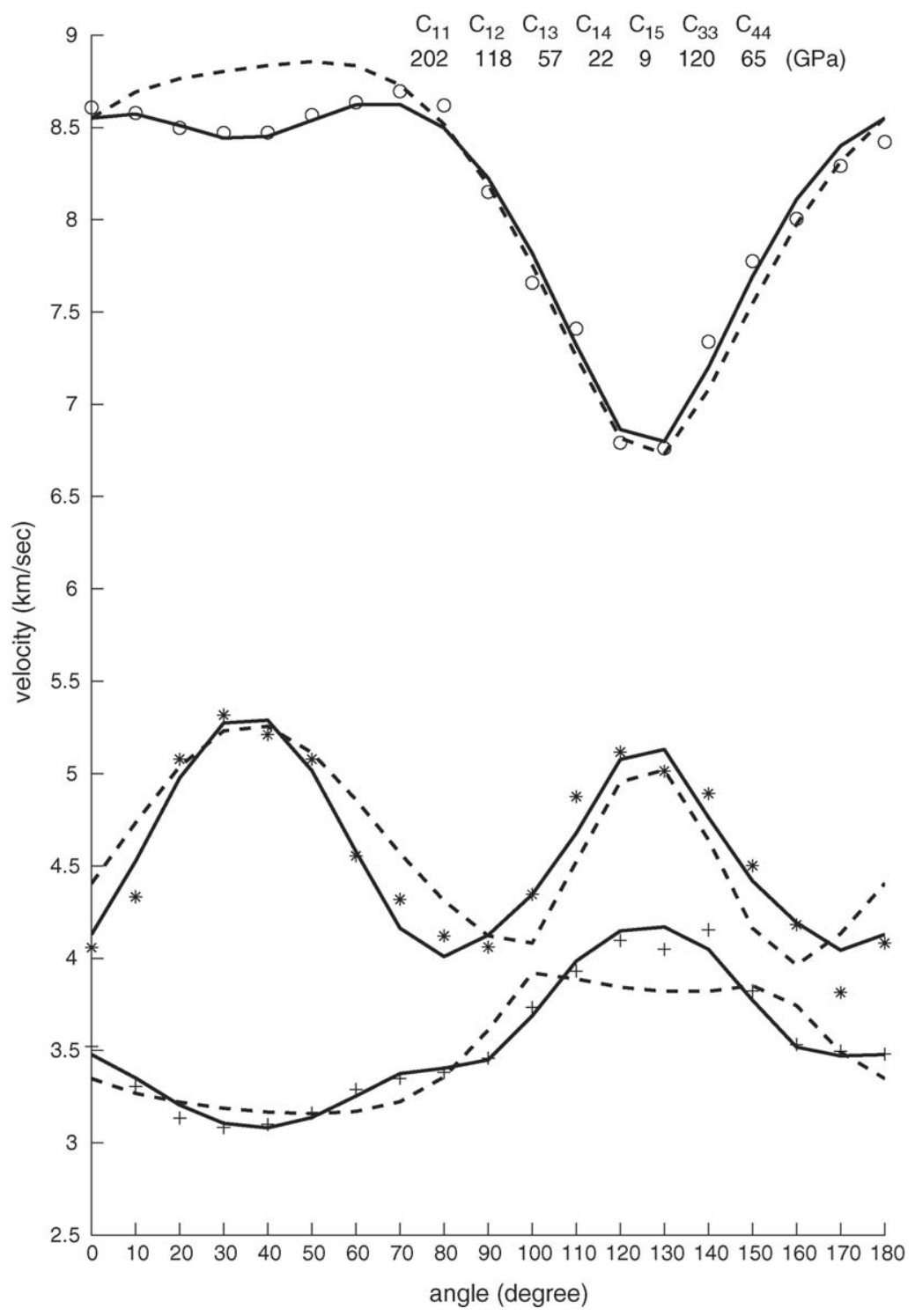

Fig. 7. The same as in Fig. 3, but for dolomite of the least misfit (0.65) group. Note the significant improvement made by local linearization (dashed lines vs. solid lines).

(0.55) are 260.3(2.6), 82.9(4.5), 59.6(3.1), (-)20.1(1.3), 153.7(4.1) and 59.7(1.4) GPa. The numbers in parentheses are uncertainties for the corresponding $C_{i j}$ 's, which were estimated by calculating the variations of misfit as a function of the specific $C_{i j}$ exclusively at the inverted solution (Fig. 6). The range of uncertainties is defined as the half width, where the differential misfit is no greater than $5 \%$ of the least misfit. In comparison with 259 , $75.6,58.8,-19.0,156$ and $54.8 \mathrm{GPa}$ as derived from an ultrasonic study (Hearmon, 1979), the elastic constants of magnesite determined by Brillouin measurements are largely in line with those derived by ultrasonic methods.
The results for dolomite are similarly shown in Table 2, except for an extra column of $C_{15}$ (column 6 ). By inspecting the results of Table 2, there are two issues needed to be clarified: (1) the solutions with similar values of misfit, $\eta$ and $\gamma$ are grouped together. While $C_{14}$ only flips sign among solutions of the same group for magnesite, both $C_{14}$ and $C_{15}$ vary in a more complicated way within the same group for dolomite. How are they related and what are the true $C_{14}$ and $C_{15}$ for dolomite? (2) Although the difference in misfit between the least $(0.65)$ and the second least $(0.66)$ groups looks minor (Figs. 7 and 8), the values of $C_{i j}$ 's between the two groups are rather different (see Table 2). 


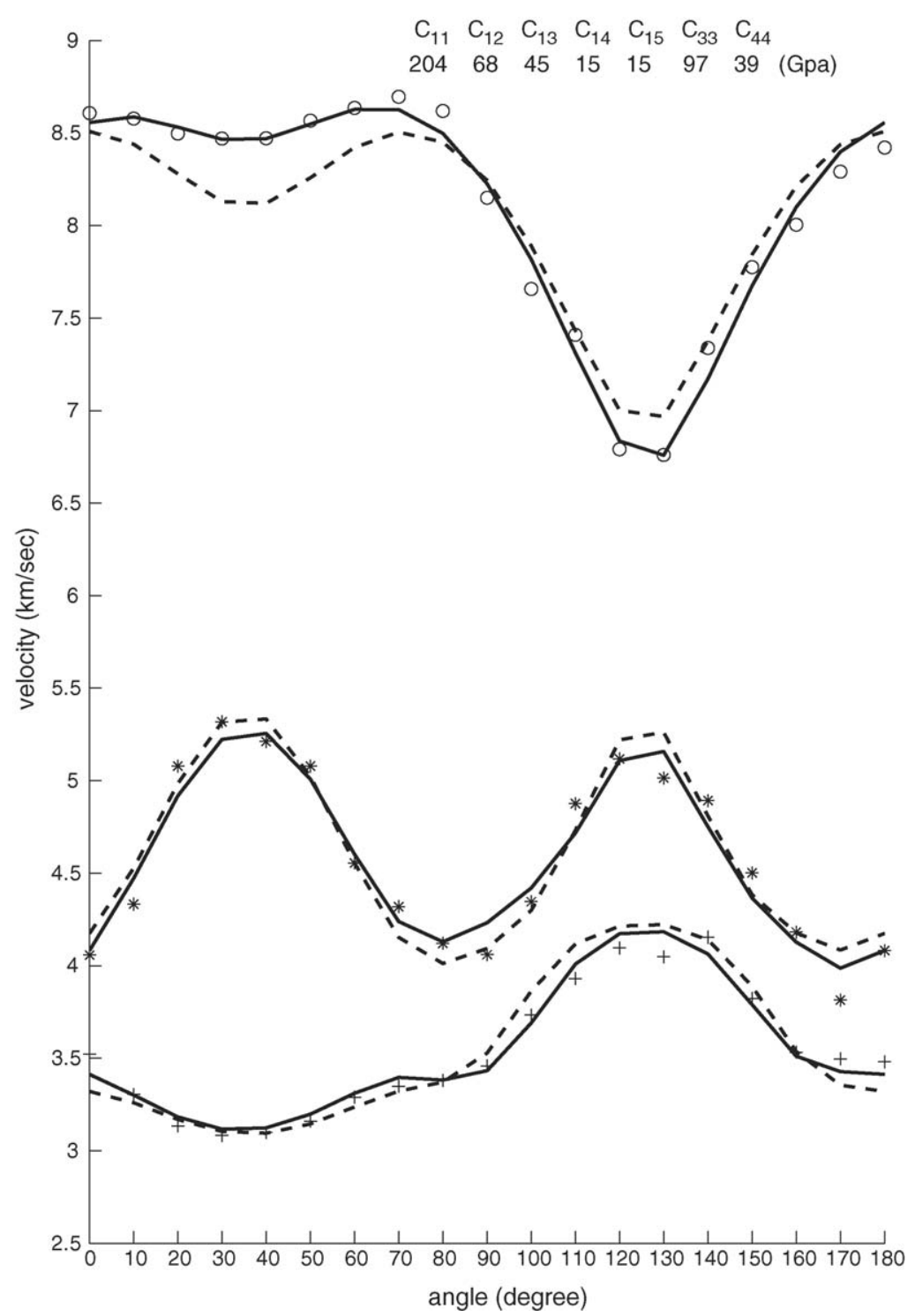

Fig. 8. The same as in Fig. 7 but for the second least misfit (0.66) group. Note that the comparable fitting between Fig. 7 and Fig. 8 , but there are substantial discrepancies in $C_{12}, C_{44}$ and probably $C_{33}$.

Which one is more appropriate for the elasticity of dolomite?

As shown in Table 2, the group of Runs \#91, 93, $104,105,152$ and 199 has a misfit $=0.65$. We arbitrarily choose the coordinates of Run \#91 as the reference. Then, the coordinates for the rest of this group derived, respectively, by rotating $15^{\circ}, 76^{\circ}, 255^{\circ}, 63^{\circ}$ and $162^{\circ}$ about the $Z$-axis are the same as those of Run \#91. The seemingly unrelated angles suggest that regardless of the angle being rotated about the $Z$-axis, a solution with the same misfit can always be determined by adapting proper $C_{14}$ and $C_{15}$. The hypothesis is tested by systematically varying $\alpha$ within the range defined by $\gamma$ with $C_{14}$ and
$C_{15}$ being the only independent variables and other $C_{i j}$ 's being fixed in the group. Upon GA application, the final solution always has the same misfit within the group, varying $C_{14}$ and $C_{15}$ according to the angle $\alpha . C_{14}$ and $C_{15}$ as a function of $\alpha$ are shown in Fig. 9 for the groups of the least and the second least misfit. According to Eq. (2), the misfit is determined by the composite differences of eigenvalues of $\Lambda_{i j}$ 's and the observed $\rho V^{2}$ at the 19 rotation angles $(\theta)$. Within a group, all solutions of $C_{i j}$ 's are the same except the variables of $C_{14}$ and $C_{15}$ and the variables of $l_{\theta}, m_{\theta}$ and $n_{\theta}$. Since $l_{\theta}, m_{\theta}$ and $n_{\theta}$ at a given rotation angle $\theta$ depend on the rotation vector, they are different with different $\alpha$ and so are the $\Lambda_{i j}$ 's produced by 

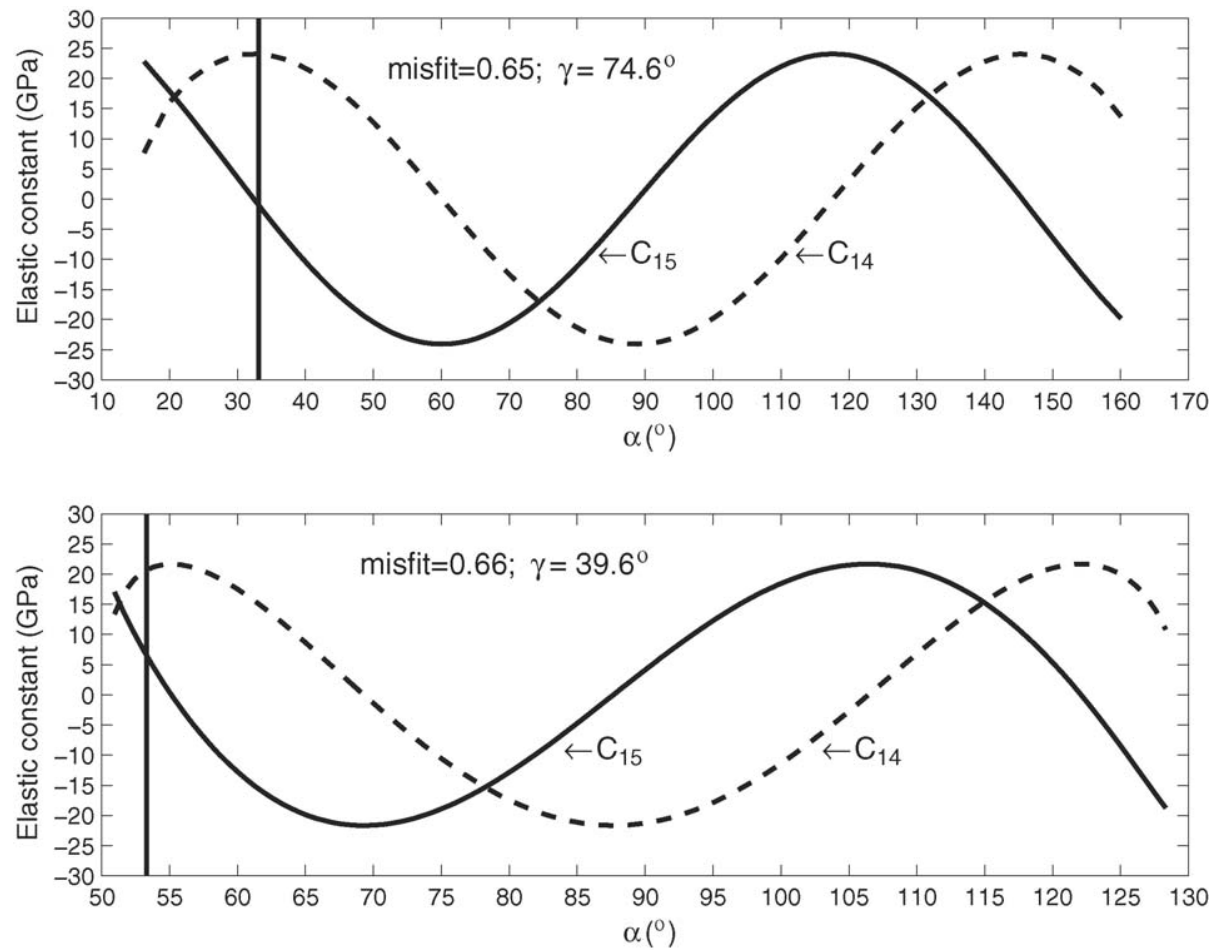

Fig. 9. $C_{14}$ and $C_{15}$ as a function of $\alpha$ for the least misfit group (upper) and the second least misfit group (lower) of dolomite. The $\alpha$ angle corresponding to the true cell rhomb (upper) and to the cleavage rhomb (lower) is marked by vertical lines.
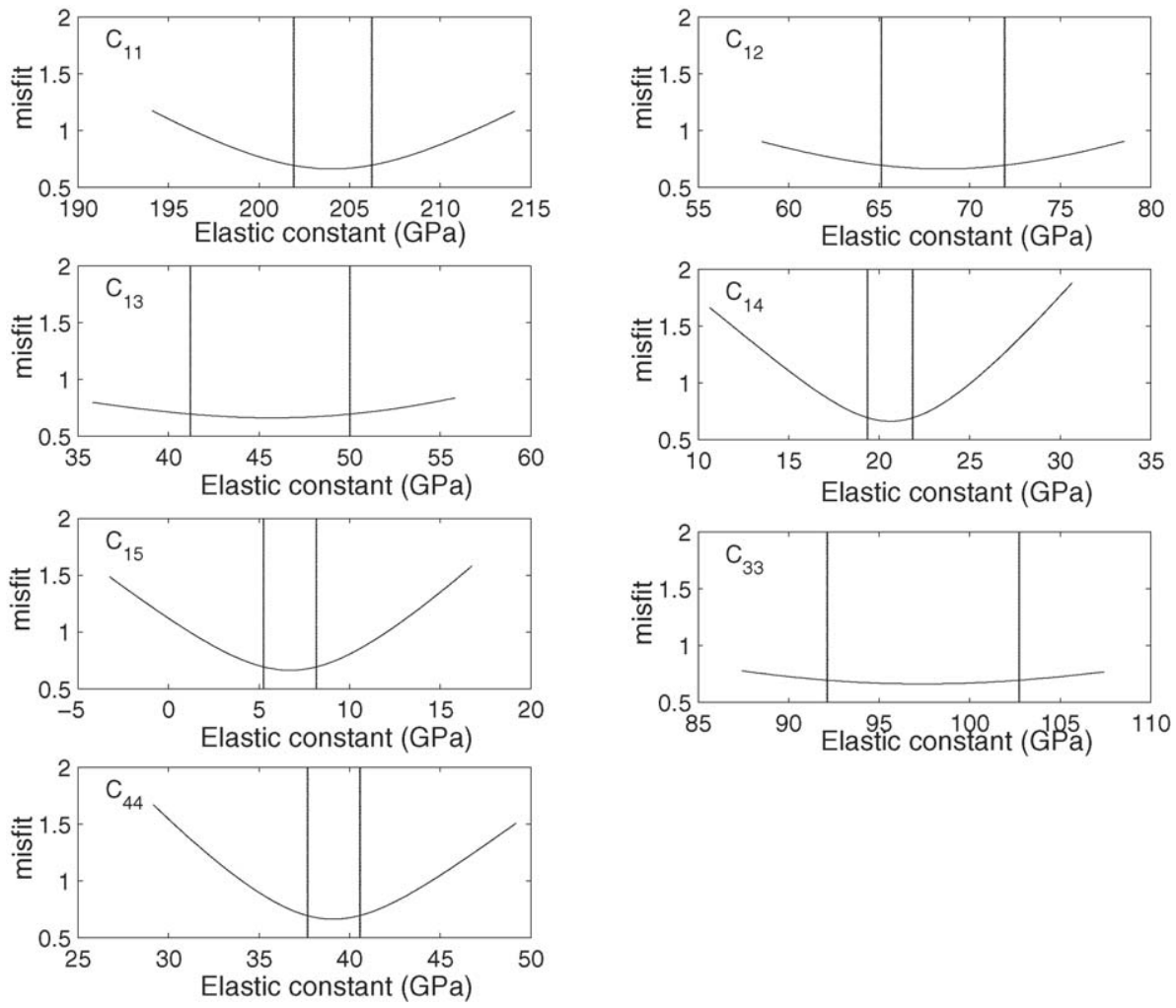

Fig. 10. The same as in Fig. 6 but for dolomite. 
their coupling with $C_{i j}$ 's. However, we find that the different eigenvalues of $\Lambda_{i j}$ 's are almost identical and yield the same misfit when results of the 19 rotation angles are summed. The implicit mathematical relationships for the non-uniqueness of $C_{14}$ and $C_{15}$ are not clear to us at present.

Since the plane of our dolomite sample is the cleavage plane, the relationships between the normal vector of the cleavage plane and the direction cosines are investigated. The calcite structure is frequently described in both (or either) the true rhombohedral unit (red in Fig. 4) and the cleavage rhombohedron (blue in Fig. 4). According to Bragg et al. (1965), the axial length and rhombohedral angle of dolomite are, respectively, $6.00 \AA$ and $47.62^{\circ}$ for the true rhomb and $6.18 \AA$ and $102.83^{\circ}$ for the cleavage rhomb. Thus, the angles for the direction cosines of the normal vector of the rhomb planes are $\left(33.1^{\circ}, 118.9^{\circ}\right.$ and $\left.75.2^{\circ}\right)$ for the true cell and $\left(53.3^{\circ}, 110.2^{\circ}\right.$ and $\left.43.6^{\circ}\right)$, for the cleavage cell, as described by the conventional Cartesian coordinates. It is noted that the $\gamma$ angles of the groups of the least and the second least misfit are $74.6^{\circ}$ and $39.6^{\circ}$ (Table 2), respectively. Accordingly, the $C_{i j}$ 's of the least misfit (0.65) group should be adopted if the plane of the true rhomb is measured and those of the second least misfit (0.66) group should be accepted if the plane of the cleavage rhomb is measured. On the basis of the known $\alpha$ angles for both planes, $C_{14}$ and $C_{15}$ are, respectively, determined to be 24.1 and $-1.0 \mathrm{GPa}$ for the true rhomb $\left(\alpha=33.1^{\circ}\right)$ and 20.6 and $6.7 \mathrm{GPa}$ for the cleavage rhomb $\left(\alpha=53.3^{\circ}\right)$ as marked by vertical lines in Fig. 9.

We tentatively adopt results of the second least misfit (0.66) group for the elasticity of dolomite because the cleavage plane was used in our measurement. Accordingly, $C_{11}, C_{12}, C_{13}, C_{14}, C_{15}, C_{33}$ and $C_{44}$ for dolomite are 204.1(2.2), 68.5(3.4), 45.8(4.4), 20.6(1.3), 6.7(1.5), 97.4(5.3) and 39.1(1.5) GPa. The uncertainties in the parentheses were derived in a similar way as those for magnesite (Fig. 10). The corresponding ultrasonic data are $205,71.0,57.4,-19.5,13.7,113$ and $39.8 \mathrm{GPa}$ for dolomite (Humbert and Plique, 1972). The results obtained between the two methods are reasonably consistent if the sign of $C_{14}$ is neglected.

\section{Conclusions}

It is concluded that the combination of GA and local linearization is an effective and innovative way of retrieving elastic constants and wave-normal directions simultaneously from Brillouin measurements. To keep the size of the initial population of GA manageable, while having statistically sufficient sampling of the model space, it is proposed that the procedure be repeated several times before a decision on the final solution is made. The results for both magnesite and dolomite indicate that the elastic constants determined by Brillouin measurements and ultrasonic methods are reasonably consistent. By analyzing the outcome of our inversion scheme, it is found that $C_{14}$ of a trigonal system could be either positive or negative, depending on choice of crystal coordinates. For a given group with a fixed misfit value, $C_{14}$ and $C_{15}$ for solutions of dolomite are not unique and depend on the angle $\alpha$. The $\gamma$ angles of the two best sets of solutions from dolomite measurements resemble those of the normal vector of planes of the true unit cell and the cleavage rhomb. The $C_{i j}$ 's corresponding to the cleavage rhomb is adopted because a cleavage plane was used in our experiments. It is anticipated that the inversion scheme developed in this study should also be applicable to crystals with lower symmetry, because no particular assumptions on symmetry are embedded within the scheme. Extension of the present scheme to crystals with lower symmetry is under way in our laboratory.

\section{Acknowledgements}

We thank C.R. Bina, C.C. Lin and two anonymous reviewers for improving the manuscript. The present work was supported by research grants from the NSC.

\section{References}

Anderson, D.L., 1989. Theory of the Earth. Blackwell Scientific Publications, Boston.

Auld, B.A., 1973. Acoustic Fields and Waves in Solids. John Wiley and Sons, New York, London, Sidney, Toronto.

Boisen Jr., M.B., Gibbs, G.V., 1985. Mathematical crystallography. Rev. Miner. 15, 1-339.

Bragg, S.L., Claringbull, G.F., Taylor, W.H., 1965. Crystal Structures of Minerals. Cornell University Press, New York.

Chen, C.C., Lin, C.C., Liu, L.G., Sinogeikin, S.V., Bass, J.D., 2001. Elasticity of single-crystal calcite and rhodochrosite by Brillouin spectroscopy. Am. Miner. 86, 1525-1529.

Dandekar, D.P., 1968a. Variation in the elastic constants of calcite with temperature. J. Appl. Phys. 39, 3694-3699.

Dandekar, D.P., 1968b. Pressure dependence of the elastic constants of calcite. Phys. Rev. 172, 873-877.

Duffy, T.S., Zha, C.S., Downs, R.T., Mao, H.K., Hemley, R.J., 1995. Elasticity of forsterite to $16 \mathrm{Gpa}$ and the composition of the upper mantle. Nature 378, 170-173.

Every, A.G., 1980. General closed-form expressions for acoustic waves in elastically anisotropic solids. Phys. Rev. B 22, 17461760.

Every, A.G., Sachse, W., 1990. Determination of the elastic constants of anisotropic solids from acoustic-wave group-velocity measurements. Phys. Rev. B 42, 8196-8205. 
Hearmon, R.F.S., 1979. The elastic constants of crystals and other anisotropic materials. In: Hellwege, K.H., Hellwege, A.M. (Eds.), Landolt-Bornstein Tables, vol. III/II. Springer-Verlag, Berlin.

Holland, J., 1975. Adaptation in Natural and Artificial Systems. The University of Michigan Press, Ann Arbor.

Houck, C.R., Joines, J.A., Kay, M.G., 1995. Technical Report No. 9509. Department of Industrial Engineering, North Carolina State University, Raleigh, NC, October 1995.

Humbert, P., Plique, F., 1972. Propriétés élastiques de carbonates rhombohédriques monocristallines: calcite, magnésite, dolomite. Comptes Rendus de 1 Academie des Sciences Paris, Series B, vol. 275. pp. 391-394.

Michalewicz, Z., 1996. Genetic algorithms + data structures $=$ evolution programs. Springer-Verlag, Berlin.

Nye, J.F., 1957. Physical Properties of Crystals. Oxford University Press, London.
Press, W.H., Teukolsky, S.A., Vetterling, W.T., Flannery, B.P., 1992. Numerical Recipes in FORTRAN: The Art of Scientific Computing, second ed. Cambridge University Press, New York.

Reeder, R.J., 1983. Crystal chemistry of the rhombohedral carbonates. Rev. Miner. 11, 1-47.

Sinogeikin, S.V., Bass, J.D., 2000. Single-crystal elasticity of pyrope and $\mathrm{MgO}$ to $20 \mathrm{GPa}$ by Brillouin scattering in the diamond cell. Phys. Earth Planet. Int. 120, 43-62.

Vo Thanh, D., Lacam, A., 1984. Experimental study of the elasticity of single crystalline calcite under high pressure (the calcite I-calcite II transition at 14.6 kbar). Phys. Earth Planet. Int. 34, 195203.

Zha, C.S., Duffy, T.S., Downs, R.T., Mao, H.-K., Hemley, R.J., 1998. Brillouin scattering and X-ray diffraction of San Carlos olivine: direct pressure determination to $32 \mathrm{GPa}$. Earth Planet. Sci. Lett. $159,25-33$ 